\title{
Preparation of IL-loaded microreactors based on polyelectrolyte microcapsules
}

\author{
Elodie Souron $^{\mathrm{a}, \mathrm{b}}$, Annie-Claude Gaumont ${ }^{\mathrm{a}}$, Karine Glinel ${ }^{\mathrm{b}}$ and Isabelle Dez ${ }^{\mathrm{a}}$ \\ a Normandie Univ., LCMT, ENSICAEN, UNICAEN, CNRS, 14000-France \\ ${ }^{\mathrm{b}}$ Institute of Condensed Matter \& Nanosciences (Bio- \& Soft Matter), Université catholique de \\ Louvain, Croix du Sud 1, B-1348 Louvain-la-Neuve, Belgium
}

contacting e-mail isabelle.dez@ensicaen.fr

Keywords: Ionic liquid; Polyelectrolyte capsule; Layer-by-Layer; Microreactor; Radical polymerisation.

Encapsulation of ionic liquids (ILs) in crosslinked polyelectrolyte microcapsules, made via layerby-layer assembly (LbL) was successfully conducted. Two different ILs were studied: 1-butyl-3methylimidazolium tetrafluoroborate $\left[\mathrm{Bmim}^{-} \mathrm{BF}_{4}\right.$ and 1-butyl-3-methylimidazolium hexafluorophosphate $\left[\mathrm{Bmim}_{\mathrm{P}} \mathrm{PF}_{6}\right.$. The polyelectrolyte microcapsules were successfully used as microcages for the synthesis of poly(methylmethacrylate) (PMMA), a non water-soluble polymer, in IL medium. Finally, the behaviour of the IL-loaded microreactors in polar and apolar solvents was evaluated. The strategies described in this study offer new routes for the preparation of microreactors incorporating IL which are of interest for many applications in the field of organic synthesis, catalysis and adsorption of active substances.

\section{Introduction}

Ionic liquids (ILs) have attracted considerable attention over the last decade. This is due to their interesting physicochemical properties, especially their low vapour pressure, as a result of which they can be considered as non-volatile organic compounds [1,2]. Accordingly, they have been regarded as environmentally friendly solvents and have been applied to a number of chemical transformations [3-5], catalytic processes [6-7] and in separation technologies [8]. Despite their interesting properties, some drawbacks are associated with the use of ILs. For instance, when they are used

as reaction media, large amounts are required, making them unattractive from an economic point of view; even if many of them are commercially available today, ILs are still expensive. Besides, they generate a large amount of waste at the end of their working lives, and their potential toxicity [9-11] and biodegradability $[12,13]$ are still to be evaluated, for most of them. Furthermore, in some cases, extraction with conventional solvents is required to separate organic products from the ionic phase, leading to some loss of the IL. 
An approach to overcome these the encapsulation of ILs allows to benefit from problems is to immobilize IL species onto solid the remarkable properties of ILs to absorb and supports while keeping their unique properties. dissolve various substances while reducing the The immobilization facilitates the interfacial drawbacks related to their high viscosity [27]. reactions and the separation processes and considerably limits the potential release of IL in the surrounding environment. Besides, it reduces the cost of the process since a smaller amount of IL is employed to perform a reaction [14-17]. A first method to immobilize ILs consists in impregnating a solid substrate on which IL forms a thin adsorbed layer. This approach is well illustrated by the current development of supported IL phase (SILP) systems, where IL layers are immobilized onto solid supports such as silica [14, 15], alumina [18] or polymers [19] and biopolymers [20-25]. SILP systems have been widely reviewed [15, 16]. Another way to immobilize ILs involves the formation of a three-dimensional hybrid network such as organosilica that percolates throughout the IL to form an ionogel (or ion gel) showing a solid-like behaviour [26]. The main drawbacks of these methods are related for the first procedure to the possible leaching of the ionic liquid and for the second to the drastic conditions used for the ionogel elaboration (long reaction time, high temperature and pressure).

The encapsulation of ILs is an attractive alternative approach to SILPs to increase the surface area to volume ratio to improve the interfacial reactions or exchanges. Moreover, Various assays essentially based on microemulsions stabilized by surfactants [28-30] or polymers [31-33], on Pickering emulsions stabilized by solid particles [34-35], or on vapour phase deposition technology [36] were explored to encapsulate ILs. However the elaboration of these systems requires complex experimental setups. Moreover, the resulting capsules showed a limited long-term stability.

Microcapsules offer a series of interesting properties such as larger specific interfacial area, semi-permeable wall and internal cavity which can be advantageously used to trap active or sensitive species. They have been widely studied for their potential applications in the pharmaceutical, agricultural, and chemical industries [37-39]. The development of microcapsules based on the Layer-by-Layer (LbL) deposition of polyelectrolytes of opposite charge onto sacrificial templates gained a considerable interest during the last fifteen years due to the versatility of the $\mathrm{LbL}$ fabrication process and the attractive properties of the resulting microcontainers [40-42]. Various compounds such as drugs [43-45], cells [46, 47], enzymes [48-50], proteins [51], DNA [52] and even organic solvents [53] have been successfully encapsulated in these microcontainers 
elaborated from synthetic polyelectrolytes or biopolymers.

Herein, we present the development of stable polyelectrolyte microcapsules incorporating IL, which can be advantageously used as microreactors for organic synthesis, catalysis or interfacial exchanges. The synthesis of a non-water soluble polymer in these microcages was investigated. Finally, the ability of these capsules to load efficiently ILs in the presence of a non-miscible solvent was tested in order to produce microcages which are of interest for heterogeneous synthesis, catalytic processes or interfacial exchanges.

Material and methods
Poly(allylamine) hydrochloride ( $\mathrm{PAH}, \mathrm{M}_{\mathrm{w}} 120$ $200 \mathrm{kDa}$ ), 1-(3-dimethylaminopropyl)-3ethylcarbodiimide hydrochloride (EDC, $>98 \%$ ) and $N$-hydroxysuccinimide (NHS, $>98 \%$ ) were purchased from Alfa Aesar. Poly(styrene sulfonate) sodium salt (PSS, $\mathrm{M}_{\mathrm{w}} 70 \mathrm{kDa}$ ) was purchased from Acros Organics. Poly(acrylic acid) solution (PAA, $\mathrm{M}_{\mathrm{w}} 100 \mathrm{kDa}, 35 \%$ wt. in $\mathrm{H}_{2} \mathrm{O}$ ) and ethylenediaminetetraacetic acid (EDTA, > 99.5\%) were purchased from Sigma Aldrich. Calcium carbonate microparticles $\left(\mathrm{CaCO}_{3},>99 \%, 6 \mu \mathrm{m}\right)$ were purchased from PlasmaChem.

1-butyl-3-methylimidazolium tetrafluoroborate $\left([\mathrm{Bmim}] \mathrm{BF}_{4}, \quad 99.9 \%\right)$ and 1-butyl-3methylimidazolium hexafluorophosphate ([Bmim] $\left.\mathrm{PF}_{6}, \quad 99.9 \%\right)$ were purchased from Solvionic and used as obtained.

Methyl methacrylate (MMA, stabilized, 99\%) and $\alpha, \alpha^{\prime}$-azoisobutyronitrile (AIBN, 98\%) were purchased from Acros Organics. MMA was purified by filtration on alumina and AIBN was recrystallized from ethanol before use. Fluorescein-labelled methylmethacrylate (fluorescein-O-MA, 97\%) was purchased from Sigma Aldrich. PAH-TRITC was synthesized as previously described [54]. Pure Milli-Q water with a specific resistivity higher than $18 \mathrm{M} \Omega . \mathrm{cm}^{2}$ was used in all experiment steps.

Elaboration of polyelectrolyte microcapsules

(PAH/PSS) microcapsules composed of 7 bilayers were prepared by alternating incubation 
of negatively charged $\mathrm{CaCO}_{3}$ microparticles $(1 \mathrm{~mL} ; 0.5 \% \mathrm{w} / \mathrm{w}$ in suspension) in PAH then PSS solution $(2 \mathrm{~g} / \mathrm{L}$ in $0.1 \mathrm{M} \mathrm{NaCl}$ for both), starting with PAH. The $\mathrm{pH}$ of the polyelectrolyte solutions was adjusted to 6.5 by addition of $0.1 \mathrm{M} \mathrm{HCl}$ or $\mathrm{NaOH}$. For each layer deposition, after $20 \mathrm{~min}$ of incubation, the coated particles were centrifuged $(3000 \mathrm{~g}$, $2 \mathrm{~min}$ ) and washed 3 times with $0.1 \mathrm{M} \mathrm{NaCl}$ to remove the non bound polyelectrolyte chains. This LbL procedure was repeated until seven bilayers were deposited. Hollow polyelectrolyte microcapsules were subsequently obtained by removing selectively the $\mathrm{CaCO}_{3}$ core by means of a $0.2 \mathrm{M}$ EDTA solution ( $\mathrm{pH}$ 5) according to the protocol described elsewhere [56-58] (Scheme 1).

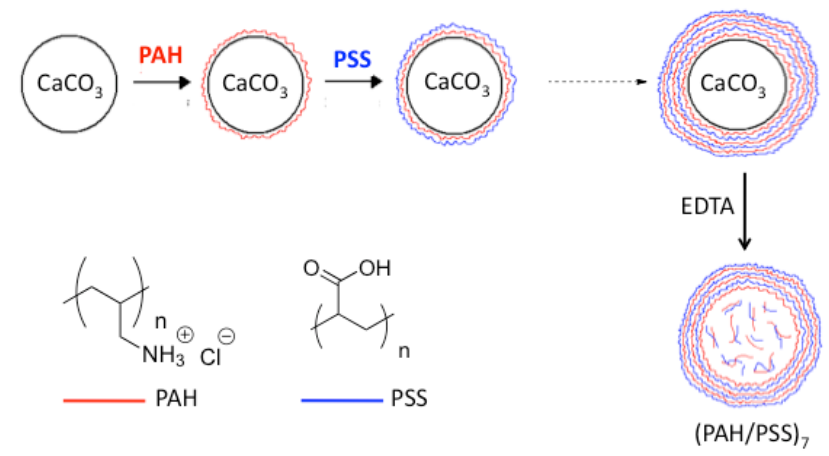

Scheme 1: (PAH/PSS $)_{7}$ microcapsules

Finally, the (PAH/PSS $)_{7}$ microcapsules were washed 3 times with pure water. Note that one layer of TRITC-labelled PAH was incorporated in the LbL sequence to visualize the microcapsules by fluorescence microscopy.

Alternatively, crosslinkable microcapsules were prepared by LbL deposition of 4 bilayers of (PAH/PSS) then 2 bilayers of (PAH/PAA) onto $\mathrm{CaCO}_{3}$ cores according to the protocol described above. The $\mathrm{pH}$ of PAH (1 mL, $2 \mathrm{~g} / \mathrm{L}$ in $0.1 \mathrm{M} \mathrm{NaCl})$ and PAA $(1 \mathrm{~mL}, 2 \mathrm{~g} / \mathrm{L}$ in $0.1 \mathrm{M}$ $\mathrm{NaCl}$ ) solutions were both fixed at 6.5.

\section{Crosslinked}

polyelectrolyte

microcapsules

Crosslinked microcapsules incorporating (PAH/PAA) bilayers were prepared according to a previously published procedure [58]. Briefly, the $\mathrm{CaCO}_{3}$-coated particles were dispersed in an EDC/NHS solution $(3 \mathrm{~mL}$ of a solution of $30 \mathrm{~g} / \mathrm{L}$ of EDC and $11 \mathrm{~g} / \mathrm{L}$ of NHS in $0.15 \mathrm{M} \mathrm{NaCl} \mathrm{pH} \mathrm{5)} \mathrm{overnight} \mathrm{at} 4^{\circ} \mathrm{C}$. The crosslinked coated particles were then washed several times with pure water and the $\mathrm{CaCO}_{3}$ core was dissolved in 0.2 M EDTA solution at pH 5 for 30 min under shaking. The resultant crosslinked

microcapsules $\left[(\mathrm{PAH} / \mathrm{PSS})_{4}(\mathrm{PAH} / \mathrm{PAA})_{2}\right]$ were washed 3 times with pure water.

Polymer synthesis in polyelectrolyte microcapsules

Microcapsules dispersed in IL were tested as microreactors for the synthesis of poly(methylmethacrylate) (PMMA). For this, hollow [(PAH/PSS $\left.)_{4}(\mathrm{PAH} / \mathrm{PAA})_{2}\right]$ crosslinked microcapsules prepared from $5 \mathrm{mg}$ of $\mathrm{CaCO}_{3}$ microparticles were dispersed in $1 \mathrm{~mL}$ of $[\mathrm{Bmim}] \mathrm{PF}_{6}$. The resulting suspension was placed in a schlenk tube and $1 \mathrm{~mL}$ more of $[\mathrm{Bmim}] \mathrm{PF}_{6}$ was added. The mixture was degassed under high vacuum for $2 h$. Then the tube was filled with nitrogen gas and MMA $(0,2 \mathrm{~mL} ; 1.87 \mathrm{mmol} ; 1$ eq. $)$ and fluorescein- 
labelled MMA (15 mg ; 0.04 mmol ; 0.02 eq.) were rapidly added. The mixture was placed in an ultrasound bath for $1 \mathrm{~h}$ at a temperature of about $10^{\circ} \mathrm{C}$ and under nitrogen atmosphere. AIBN (3 mg ; $0.019 \mathrm{mmol} ; 0.01$ eq.) initiator was subsequently added into the tube which was placed for $5 \mathrm{~min}$ in an ultrasound bath then heated at $65^{\circ} \mathrm{C}$ for $4 \mathrm{~h}$ under nitrogen atmosphere. At the end of the reaction, the heterogeneous mixture was dispersed in $10 \mathrm{~mL}$ of acetone and the capsules were recovered by centrifugation. Five washing cycles with acetone were performed to remove the nonencapsulated PMMA chains.

The capsules were observed with an epifluorescence microscope in a water/acetone $(50 / 50 \mathrm{v}: \mathrm{v})$ mixture.

Encapsulation of ionic liquid in polyelectrolyte microcapsules

Hollow microcapsules prepared from $5 \mathrm{mg}$ of $\mathrm{CaCO}_{3}$ core particles, were centrifuged $(3000 \mathrm{~g}$, $5 \mathrm{~min}$ ) and the water supernatant was replaced by $150 \mu \mathrm{L}$ of either $[\mathrm{Bmim}] \mathrm{PF}_{6}$ or $[\mathrm{Bmim}] \mathrm{BF}_{4}$. The residual traces of water were removed with a centrifugal SpeedVac evaporator for $1 \mathrm{~h}$. The resulting suspension was filtered through a polycarbonate cyclopore membrane (porosity $400 \mathrm{~nm}$; It4ip, Belgium). The ILloaded microcapsules adsorbed on the polycarbonate membrane were rapidly rinsed with $4 \times 0.5 \mathrm{~mL}$ of a solvent non miscible with IL to drag the excess of the non loaded ionic liquid. Pure water and isopropanol were used as non miscible solvents for $\left[\mathrm{Bmim}_{\mathrm{P}}\right] \mathrm{PF}_{6}$ and $[\mathrm{Bmim}] \mathrm{BF}_{4}$, respectively. The microcapsules were finally recovered by immersing the polycarbonate membrane into a beaker containing $2 \mathrm{~mL}$ of a non-miscible solvent and placed for $1 \mathrm{~min}$ in an ultrasound bath.

\section{Epifluorescence Microscopy}

The samples labelled with TRITC or fluorescein were observed using a LEICA DMR fluorescent microscope respectively with a TEXAS-RED filter or a $\mathrm{H}_{3}$ filter. Pictures were taken using an ORCA AG Hamamatsu digital charged-coupled device (CCD) camera driven by Wasabi 2.0 software.

Scanning Electron Microscopy - Energy Dispersive Spectroscopy (SEM-EDS)

Few drops of capsule suspension were deposited onto a glass slide and the solvent was gently dried. The resulting samples were coated with a $20-n m$ thick film of gold (Fine coat JFC 1100). Analyses were performed using a scanning electron microscope (SEM) from ZEISS SUPRA 55 equipped with INLENS and SE2 detectors and coupled with energy dispersive spectroscopy (EDS). Magnifications x 3000 and x 5000 were used for imaging with an accelerating potential of $20 \mathrm{kV}$.

\section{Results and discussion}

Preparation of PE microcapsules stable in IL

Polyelectrolyte capsules have been previously used as reaction cages in water to synthesize 
polymers, notably polyelectrolytes [59]. According to this "ship in bottle" methodology, the small monomers diffuse through the polyelectrolyte membrane then polymerise toThis technique allowed producing capsules with permanently modified interior. Such microcontainers are of interest in various fields such as chemical catalysis or drug release as they required low amounts of reagents, and the confiment of the media and reagents afforded a more rapid heat exchange and mass transfer as compared to conventional, larger scale systems. Here, we explore the possibility to use polyelectrolyte microcapsules as microreactors in IL environment to perform chemical synthesis. Such microreactors combine the advantages of the microcapsules and the relevant properties of ILs. In a first approach, the non-crosslinked polyelectrolyte capsules obtained after core dissolution were dispersed directly in ILs to test their stability in such solvents. Experimentally, hollow microcapsules composed of 7 bilayers of (PAH/PSS) [60] were dispersed in $\left[\mathrm{Bmim}^{\mathrm{P}} \mathrm{PF}_{6}\right.$, IL which is known to have a hydrophobic character. Observations performed by fluorescence microscopy on PAHTRITC labelled capsules showed that the microcontainers collapsed to form large aggregates as soon they were dispersed in IL (Figure 1). Zoom-in image (Figure 1 inset)
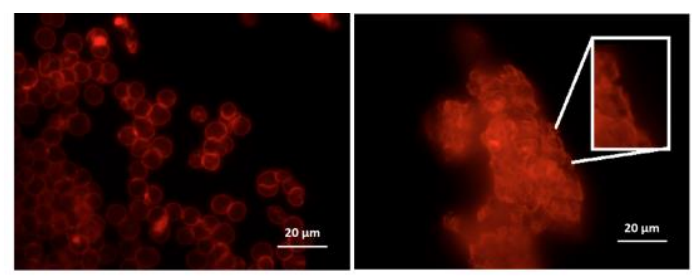

revealed that polyelectrolyte walls merged.

Figure 1 Fluorescent microscopy images (x 100) of $(\mathrm{PAH} / \mathrm{PSS})_{7}$ microcapsules labelled with PAH-TRITC and dispersed in water (left) and in $\left[\mathrm{Bmim}_{\mathrm{P}} \mathrm{PF}_{6}\right.$ (right)

Similar results were observed for the $(\mathrm{PAH} / \mathrm{PSS})_{7}$ capsules dispersed in the more hydrophilic IL [Bmim] $\mathrm{BF}_{4}$ (Figure 2). These results clearly evidenced that non-crosslinked microcapsules were not stable in the presence of ILs, independently of its properties (hydrophilic or hydrophobic). This behaviour may be attributed to the electrostatic interactions between IL ions and charged groups of polyelectrolyte chains that induced a disruption of the polyelectrolyte complex. To evidence this feature, a certain amount of water was added in the $[\mathrm{Bmim}] \mathrm{BF}_{4} / \mathrm{capsule}$ suspension to increase the dissociation of water-soluble IL ions. As seen in Figure 2, the addition of water impacted more significantly the integrity of the $(\mathrm{PAH} / \mathrm{PSS})_{7}$ capsules.

a)
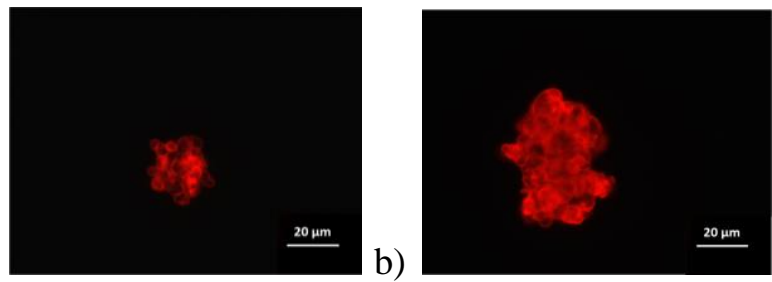

c)
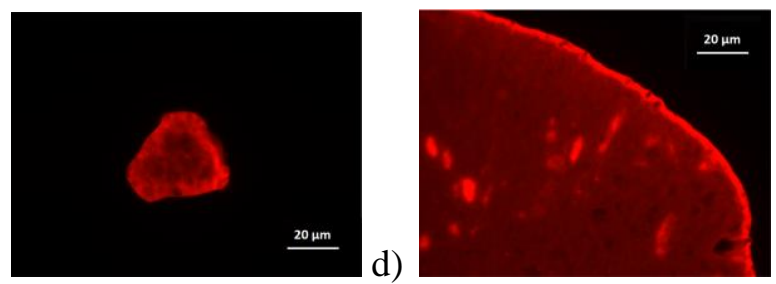
Figure 2 Fluorescent microscopy images of $(\mathrm{PAH} / \mathrm{PSS})_{7}$ microcapsules labelled with PAHTRITC and dispersed in a) pure $\left[\mathrm{Bmim}_{\mathrm{B}} \mathrm{BF}_{4}\right.$ and in $[\mathrm{Bmim}] \mathrm{BF}_{4} /$ water mixtures of different ratio (v/v): b) 70/30 c)50/50 d)30/70

For instance, in presence of 70 vol \% of water, a complete destruction of the capsules was observed (Figure 2-d). Therefore, ionic components of ILs disrupted the polyelectrolyte complex. They partially screened the electrostatic interactions between polyelectrolyte chains. This effect was more pronounced in presence of water due to the dissociation of IL ions. The ability of IL ions to disrupt a polyelectrolyte complex leads to an unstable behaviour of the non-cross-linked microcapsules in Ils and IL/water mixture.

To improve the stability of the polyelectrolyte microcontainers in the presence of ILs, capsules incorporating crosslinked layers were tested. Experimentally, $\mathrm{CaCO}_{3}$ cores coated by 4 (PAH/PSS) bilayers were functionalized by two additional (PAH/PAA) bilayers. Crosslinking of the (PAH/PAA) sequence was achieved in water in presence of EDC/NHS according to the protocol described elsewhere [61]. Finally, the $\mathrm{CaCO}_{3}$ core was destroyed in acidic conditions as described in the experimental section. In order to test the stability of the crosslinked wall towards ILs, the capsules were dispersed in $[\mathrm{Bmim}] \mathrm{BF}_{4}$ or $[\mathrm{Bmim}] \mathrm{PF}_{6}$ then observed by fluorescence microscopy.
As shown in Figure 3, the integrity of the hollow capsules was preserved, even after $48 \mathrm{~h}$ in $[\mathrm{Bmim}] \mathrm{BF}_{4}$. Similar results were obtained in the more hydrophobic IL $\left[\mathrm{Bmim} \mathrm{PF}_{6}\right.$ (results not shown).

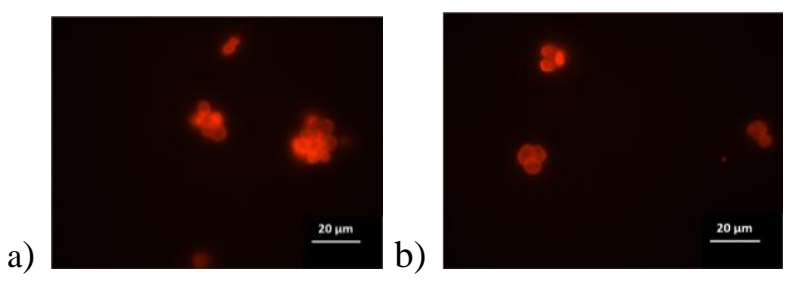

Figure 3 Cross-linked (PAH/PSS $)_{4}(\mathrm{PAH} / \mathrm{PAA})_{2}$ microcapsules labelled with PAH-TRITC and dispersed in $[\mathrm{Bmim}] \mathrm{BF}_{4}$ after different times : (a) $\mathrm{t}=0$ and (b) $\mathrm{t}=48 \mathrm{~h}$

PE Microcapsules used as microreactors for the synthesis of a non-water-soluble polymer The $\left[(\mathrm{PAH} / \mathrm{PSS})_{4}(\mathrm{PAH} / \mathrm{PAA})_{2}\right] \quad$ crosslinked capsules were subsequently tested as microreactors to synthesize a non-water soluble PMMA polymer in IL medium. Radical polymerisation was selected since ILs are known to be good solvents to achieve such reactions [62-64]. Experimentally, the protocol developed by Dähne et al. [59] was adapted. Briefly, the microcapsules were dispersed in $[\mathrm{Bmim}] \mathrm{PF}_{6}$ in presence of the MMA monomer. The AIBN initiator was rapidly added into the reactive mixture which was stirred at $65^{\circ} \mathrm{C}$ for 4h under a controlled atmosphere. At the end of the reaction, the PMMA-loaded capsules were recovered by centrifugation and washed with acetone to remove the non encapsulated PMMA chains formed in solution. Note that $2 \%$ of 101 
fluorescein-labelled MMA monomer was added in the reaction mixture to visualize the formation of PMMA chains by fluorescence microscopy.

Microscopy observations performed on the microcapsules clearly revealed that labelled PMMA chains were entrapped inside the capsules, having a core radius between 8 and 4 $\mu \mathrm{m}$, and that kept their spherical shape (Figure 4).

a)

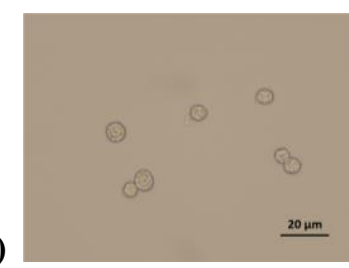

b)

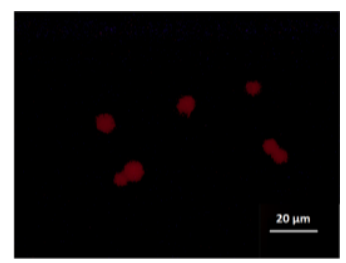

Figure 4 Microscopy images showing fluorescein-labelled PMMA encapsulated into $\left[(\mathrm{PAH} / \mathrm{PSS})_{4}(\mathrm{PAH} / \mathrm{PAA})_{2}\right] \quad$ crosslinked microcapsules: a) optical micrograph and b) fluorescence image. The capsules are dispersed in water/acetone $(50 / 50 \mathrm{v} / \mathrm{v})$ mixture

These conditions afforded the synthesis of PMMA with high molar weight (> 9.9.10 g.mol ${ }^{-1}$ ) [65]. The molar mass of the PMMA chains was large enough to avoid any release through the capsule wall. Therefore, the polymer chains remained efficiently trapped into the microcontainers. Such loaded capsules encapsulating macromolecules non-soluble or partially soluble in water should be of interest to trap hydrophobic compounds in various media. Indeed, these solvent responsive materials, which swell in organic solvent and precipitate in water, should be used notably to entrap catalytic species or to remove hydrophobic substrates from reactive organic media.

\section{Preparation and stability of IL-loaded} microcapsules dispersed in various solvents

Following the successful fabrication of polyelectrolyte microcapsules stable towards ILs, the possibility to prepare microcontainers entrapping permanently IL was explored in order to fabricate IL-loaded microreactors. Two different systems based on IL-loaded microcapsules dispersed in water or isopropanol were tested. The procedure followed for the IL encapsulation consisted in dispersing the crosslinked microcapsules into the IL to achieve its diffusion into the capsule interior. After loading, the capsules were recovered by filtration then dispersed in water or isopropanol. To evaluate the stability of the IL-loaded capsules, they were observed by SEM coupled with EDS after a given time. Indeed, due to their very low vapour pressure, ILs can be observed under vacuum condition. A first test was performed with capsules loaded with $[\mathrm{Bmim}] \mathrm{BF}_{4}$ and dispersed in water which is miscible with $\left[\mathrm{Bmim}^{-} \mathrm{BF}_{4}\right.$. 

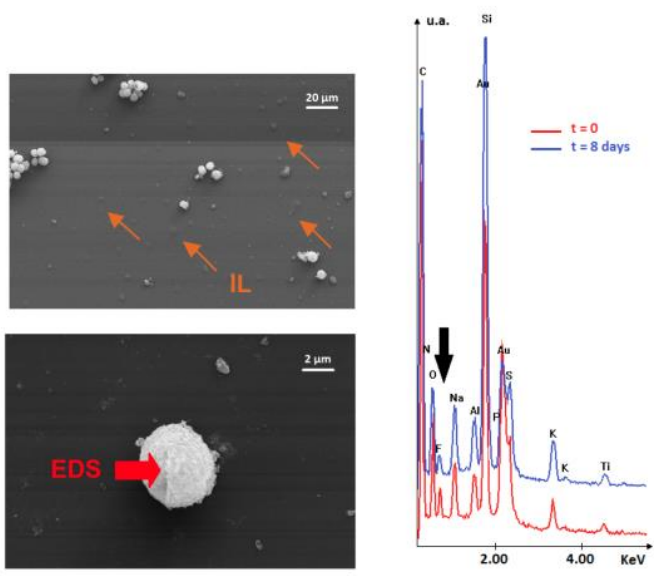

Figure 5 (Left) SEM image of [Bmim] $] \mathrm{BF}_{4}$-loaded microcapsules; (right) EDS spectra performed after different immersion times of the capsules into isopropanol.

In this case, a total release of the IL was observed showing a total permeability of the polyelectrolyte capsules in this medium. The loaded capsules were then dispersed in isopropanol which is non-miscible with the IL. SEM image displayed in Figure 5 showed that capsules kept their spherical shape even after IL loading with a core radius higher than those of the empty capsules. Moreover, EDS spectrum recorded on a capsule (Figure 5), revealed a fluorine peak centred at $0.677 \mathrm{keV}$ which testified for the presence of $[\mathrm{Bmim}] \mathrm{BF}_{4}$.

However, the amount of loaded IL could not be determined since EDS is not a quantitative technique. Moreover, it has to be noticed that few droplets of free $[\mathrm{Bmim}] \mathrm{BF}_{4}$ surrounding the capsules were also detected by SEM-EDS analysis. These droplets may be attributed to a partial release of IL during the sample preparation or to an incomplete washing of the capsules. To definitely conclude about the stability of the loading, the ILloaded capsules dispersed in isopropanol were recovered by centrifugation then rinse with pure isopropanol and analysed by SEM-EDS. The EDS spectra recorded on the capsules confirmed the presence of a fluorine peak related to $[\mathrm{Bmim}] \mathrm{BF}_{4}$ and SEM observation did not show the presence of IL droplets around the capsules (Figure 6) as observed before. Altogether these results proved that $[\mathrm{Bmim}] \mathrm{BF}_{4}$ is well entrapped into the capsules that have a radius of the core between 4-6 $\mu \mathrm{m}$; and not simply adsorbed onto the capsule wall.

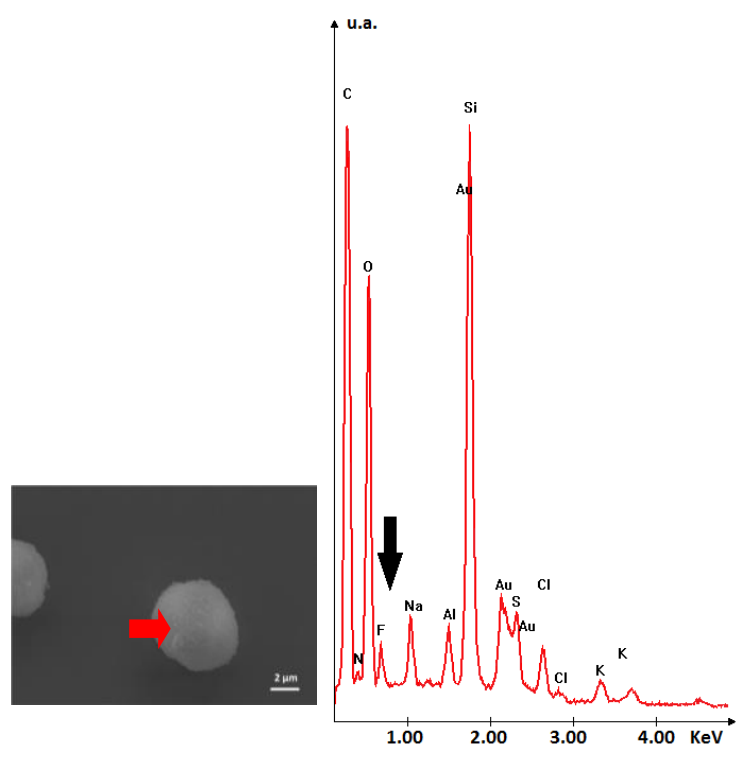

Figure 6 SEM-EDS analysis of [Bmim] $\mathrm{BF}_{4}$-loaded microcapsules washed 2 times with isopropanol.

Similar experiments were performed with capsules loaded with a more hydrophobic IL 
such as $\left[\mathrm{Bmim} \mathrm{PF}_{6}\right.$ and dispersed in water which is non miscible with the IL. Interestingly, a rapid leaching of $\mathrm{IL}$ in the aqueous phase was observed. EDS analysis revealed that the IL is totally removed from the capsule after only one washing with water (result not shown). This result may be explained considering the hydrophilic nature of the polyelectrolyte wall of the capsules. In hydrophilic medium, the polyelectrolyte film swelled to form a semipermeable barrier through which the IL molecules could rapidly diffuse and release. In contrast, in presence of isopropanol which is a non solvent of polyelectrolyte chains, the LbL film collapsed to form a dense membrane limiting the release of loaded IL in the surrounding environment.

Therefore, the entrapment of ILs into polyelectrolyte microcapsules was properly achieved with a hydrophilic IL in isopropanol.

\section{Conclusions}

Polyelectrolyte microcapsules have been extensively used previously to trap various active substances in aqueous solution. However, their non stability in other solvents limits their potential applications. By performing a partial crosslinking of the polyelectrolyte wall, we prepared microcapsules stable in highly polar ionic liquid phases. We demonstrated that these microreactors incorporating IL can be successfully used to synthesize non watersoluble polymers such as PMMA. Moreover, the stability of the IL-loaded microreactors in different media was evaluated: a rapid release of the ILs in water was observed whereas no release of the IL occurred for $[\mathrm{Bmim}] \mathrm{BF}_{4}$ loaded microcapsules in isopropanol. It was concluded that the release behavior of the IL is mainly related to the nature of the surrounding medium. This feature may contribute to apply the IL loaded microcapsules as solvent responsive materials, which release the IL in aqueous medium but not in organic medium, for catalysis application or liquid-liquid extraction.

The IL-loaded microreactors offer the advantage to associate the ability of the microcapsules to be used as microcontainer with the remarkable physicochemical properties of ionic liquids. Moreover, the IL-loaded capsules are more stable and easier to implement than those developed previously via microemulsion processes as they require the use of any surfactant. Therefore, these microreactors are of great interest for organic synthesis, catalysis or liquid-liquid extraction. The liquid-core capsules constitute an alternative to SILP systems to perform interfacial reactions, while benefiting from the confiment effect of the IL media in term of low overconcentration of reactives or active substances and overheating of the system, and from the presence of the microcage to trap these active substances.

\section{Acknowledgements}

E. Ferain and C. Douchamps (from UCL, Belgium) and J. Lecourt (from UCBN, 
CRISMAT) are thanked for their technical assistance with SEM measurements. A.-C. Faber (from UCL, ISV) is thanked for her technical assistance with epifluorescence microscopy. E.S. benefits from a financial support by French CNRS and Région Basse Normandie within the frame of "Institut Normand de Chimie Moléculaire, Médicinale et Macromoléculaire ". KG benefits from a "M.I.S - Mandat ULYSSE" from the Belgium F.R.S. FNRS.

\section{References}

[1] Wasserscheid P, Welton T. Ionic liquids in synthesis. Wiley-VCH, Weinheim 2008

[2] Angell C, Ansari Y, Zhao Z. Ionic liquids: Past, present and future. Faraday Discuss 2012;154:9-27

[3] Martins MAP, Frizzo CP, Moreira DN, Zanatta N, Bonacorso HG. Ionic liquids in heterocyclic synthesis. Chem Rev 2008;108:2015-2050

[4] Singh R, Sharma M, Mamgain R, Rawat DS. Ionic liquids: A versatile medium for palladium-catalyzed reactions. J Braz Chem Soc 2008;19:357-379

[5] Winkel A, Reddy PVG, Wilhelm R. Recent advances in the synthesis and application of chiral ionic liquids. Synthesis 2008;999-1016

[6] Dominguez de Maria P. "Nonsolvent" applications of ionic liquids in biotransformations and organocatalysis. Angew Chem Int Ed 2008;47:6960-6968

[7] Hallett JP, Welton T. Room-temperature ionic liquids: solvents for synthesis and catalysis. 2 . Chem Rev 2011;111:3508-3576

[8] Han D, Row KH. Recent applications of ionic liquids in separation technology. Molecules 2010;15:2405-2426

[9] Jastorff B, Störmann R, Ranke J, Mölter K, Stock F, Oberheitmann B, Hoffmann W, Hoffmann J, Nüchter M, Ondruschka B, Filser J. How hazardous are ionic liquids? Structure-activity relationships and biological testing as important elements for sustainability evaluation. Green Chem 2003;5:136-142

[10] Zhao D, Liao Y, Zhang Z. Toxicity of ionic liquids. Clean 2007;35:42-48

[11] Zheng Z, Zhigang C, Minhua Z, Zhenxin G.

Toxicity, biodegradability and design and synthesis of green ionic liquids. Chinese J Org Chem 2009;29:672-680

[12] Stolte S, Abdulkarim S, Arning J, Blomeyer-Nienstedt AK, Bottin-Weber U, Matzke M, Ranke J, Jastorff B, Thöming J. Primary biodegradation of ionic liquid cations, identification of degradation products of 1-methyl-3-octylimidazolium chloride and electrochemical wastewater treatment of poorly biodegradable compounds. Green Chem. 2008;10:214224

[13] Harjani JR, Farrell J, Garcia MT, Singer RD, Scammells PJ. Further investigation of the biodegradability of imidazolium ionic liquids. Green Chem 2009;11:821-829

[14] Valkenberg MH, de Castro C, Holderich WH. Immobilisation of ionic liquids on solid supports. Green Chem 2002;4:88-93

[15] Mehnert CP, Supported ionic liquid catalysis. Chem Eur J 2005;11:50-56

[16] Riisager A, Fehrmann R, Haumann M, Wasserscheid P. Supported ionic liquids: versatile reaction and separation media. Top Catal 2006;40:91-102

[17] Gua Y, Li G. Ionic liquids-based catalysis with solids: state of the art. Adv Synth Catal 2009;351:817-847

[18] Hagiwara H, Ko KH, Hoshi T, Suzuki T. Supported ionic liquid catalyst (Pd-SILC) for highly efficient and recyclable Suzuki-Miyaura reaction. Chem Commun 2007;2838-2840

[19] Wolfson A, Vankelecom IFJ, Jacobs PA. Co-immobilization of transition-metal complexes and ionic liquids in a polymeric support for liquid-phase hydrogenations. Tetrahedron Lett 2003;44:1195-1198 
[20] Baudoux J, Perrigaud K, Madec PJ, Gaumont AC, Dez I. Development of new SILP catalysts using chitosan as support. Green Chem 2007;9:1346-1351

[21] Moucel R, Perrigaud K, Goupil JM, Madec PJ, Marinel S, Guibal E, Gaumont AC, Dez I. Importance of the conditioning of the chitosan support in a catalystcontaining ionic liquid phase immobilised on chitosan: The palladium-catalysed allylation reaction case. Adv Synth Cat 2010;352:433-439

[22] Clousier N, Moucel R, Naik P, Madec PJ, Gaumont AC, Dez I. Catalytic materials based on catalysts containing ionic liquid phase supported on chitosan or alginate: Importance of the support. Comptes Rendus Chimie 2011;14:680-684

[23] Vincent T, Guibal E. Chitosan-supported palladium catalyst. 5. nitrophenol degradation using palladium supported on hollow chitosan fibers. Environ Sci Technol 2004;38:4233-4240

[24] Guibal E, Vincent T, Jouannin C. Immobilization of extractants in biopolymer capsules for the synthesis of new resins: a focus on the encapsulation of tetraalkyl phosphonium ionic liquids. J Mater Chem 2009;19:8515-8527

[25] Jouannin C, Dez I, Gaumont AC, Taulemesse JM, Vincent T, Guibal E. Palladium supported on alginate/ionic liquid highly porous monoliths: Application to 4-nitroaniline hydrogenation. Appl Catal B: Environ 2011;103:444-452

[26] Le Bideau J, Viau L, Vioux A. Ionogels, ionic liquid based hybrid materials. Chem Soc Rev 2011;40:907-925

[27] Earle MJ, Seddon KR. Ionic liquids. Green solvents for the future. Pure Appl Chem 2000;72:13911398

[28] Gao HX, Li JC, Han BX, Chen WN, Zhang JL, Zhang R, Yan DD. Microemulsions with ionic liquid polar domains. Phys Chem Chem Phys 2004;6:2914-2916

[29] Gao Y, Li N, Zheng LQ, Zhao XY, Zhang SH, Han BX, Hou WG, Li GZ. A cyclic voltammetric technique for the detection of micro-regions of
bmimPF6/Tween 20/H2O microemulsions and their performance characterization by UV-Vis spectroscopy. Green Chem 2006;8:43-49

[30] Zheng Y, Eli W. Study on the Polarity of bmimPF6/Tween80/toluene Microemulsion Characterized by UV-Visible Spectroscopy. J Dispersion Sci Technol 2009;30:698-703

[31] Yang WW, Lu YC, Xiang ZY, Luo GS. Monodispersed microcapsules enclosing ionic liquid of 1butyl-3-methylimidazolium hexafluorophosphate. React Funct Polym 2007;67:81-86

[32] Gao H, Xing J, Xiong X, Li Y, Li W, Liu Q, $\mathrm{Wu} Y$, Liu $\mathrm{H}$. Immobilization of Ionic Liquid [BMIM][PF6] by Spraying Suspension Dispersion Method. Ind Eng Chem Res 2008;47:4414-4417

[33] Xiang ZY, Lu YC, Zou Y, Gong XC, Luo GS. Preparation of microcapsules containing ionic liquids with a new solvent extraction system. React Funct Polym 2008;68:1260-1265

[34] Ma H, Dai LL. Particle Self-Assembly in Ionic Liquid-in-Water Pickering Emulsions. Langmuir 2011;27:508-512

[35] Binks BP, Dyab AKF, Fletcher PDI. Novel emulsions of ionic liquids stabilised solely by silica nanoparticles. Chem Commun 2003;2540-2541

[36] Bradley LC, Gupta M. Encapsulation of Ionic Liquids within Polymer Shells via Vapor Phase Deposition. Langmuir 2012;28:10276-10280

[37] Saito T, Torii S. Microcapsule for adsorption and recovery of cadmium(II) ion. Sep Sci Technol 2002;37:77-87

[38] Shchukin DG, Patel AA, Sukhorukov GB, Lvov YM. Nanoassembly of biodegradable microcapsules for DNA encasing. J Am Chem Soc 2004; 126:3374-3375

[39] Ye S, Wang C, Liu X, Tong Z, Ren B, Zeng F. New loading process and release properties of insulin from polysaccharide microcapsules fabricated through layer-by-layer assembly. J Controlled Release 2006;112:79-87 
[40] Sukhorukov GB, Fery A, Möhwald H. Intelligent micro- and nanocapsules. Prog Polym Sci 2005;30:885-897

[41] Peyratout CS, Dähne L. Tailor-made polyelectrolyte microcapsules: From multilayers to smart containers. Angew Chem Int Ed 2004;43:3762-3783

[42] Sukhorukov GB, Rogach AL, Zebli B, Liedl T, Skirtach AG, Koehler K, Antipov AA, Gaponik N, Susha AS, Winterhalter M, Parak WJ. Nanoengineered polymer capsules: tools for detection, controlled delivery, and site-specific manipulation. Small 2005;1:194-200

[43] De Koker S, Hoogenboom R, De Geest BG. Polymeric multilayer capsules for drug delivery. Chem Soc Rev 2012;41:2867-2884

[44] Anandhakumar S, Debapriya M, Nagaraja V, Raichur AM. Polyelectrolyte microcapsules for sustained delivery of water-soluble drugs. Mater Sci Eng C 2011;31:342-349

[45] Shchukina EM, Shchukin DG. LbL coated microcapsules for delivering lipid-based drugs. Adv Drug Delivery Rev 2011;63:837-846

[46] Neu B, Voigt A, Mitlöhner R, Leporatti S, Gao CY, Donath E, Kiesewetter H, Möhwald H, Meiselman HJ, Baumler H. Biological cells as templates for hollow microcapsules. J Microencapsulation 2001;18:385-395

[47] Fakhrullin RF, Minullina RT. Hybrid cellular-inorganic core-shell microparticles: encapsulation of individual living cells in calcium carbonate microshells. Langmuir 2009;25:6617-6621

[48] Zhao ZX, Wang XS, Qin X, Chen Q, Anzai JI. Enzyme microcapsules with substrate selective permeability constructed via layer-by-layer polyelectrolyte self-assembly. Mater Sci Eng, C 2012;32:569-573

[49] Kazakova LI, Shabarchina LI, Sukhorukov GB. Co-encapsulation of enzyme and sensitive dye as a tool for fabrication of microcapsule based sensor for urea measuring. Phys Chem Chem Phys 2011;13:11110-11117
[50] Nayak SR, McShane MJ. Encapsulation of peroxidase by polymerizing acrylic acid monomers in "clean" polyelectrolyte microcapsules. J Biomed Nanotechnol 2007;3:170-177

[51] De Temmerman ML, Demeester J, De Vos F, De Smedt SC. Encapsulation performance of layer-bylayer microcapsules for proteins. Biomacromolecules 2011;12:1283-1289

[52] Wang Z, Qian L, Wang X, Yang F, Yang X. Construction of hollow DNA/PLL microcapsule as a dual carrier for controlled delivery of DNA and drug. Colloids Surf, A 2008;326:29-36

[53] Moya S, Sukhorukov GB, Auch M, Donath E, Möhwald H. Microencapsulation of organic solvents in polyelectrolyte multilayer micrometer-sized shells. J Colloid Interface Sci 1999;216:297-302

[54] Cordeiro A, Coelho M, Sukhorukov G, Dubreuil F, Möhwald H. Effect of shear stress on adhering polyelectrolyte capsules. J Colloid Interface Sci 2004;280:68-75

[55] Andreeva DV, Gorin DA, Möhwald H, Sukhorukov GB. Novel type of self-assembled polyamide and polyimide nanoengineered shellssfabrication of microcontainers with shielding properties. Langmuir 2007;23:9031-9036

[56] Wang Z, Zhu H, Li D, Yang X. Preparation and application of single polyelectrolyte microcapsules possessing tunable autofluorescent properties. Colloids Surf, A 2008;329:58-66

[57] Volodkin DV, Petrov AI, Prevot M, Sukhorukov GB. Matrix polyelectrolyte microcapsules: new system for macromolecule encapsulation. Langmuir 2004;20:3398-3406

[58] Richert L, Bouldemais F, Lavalle P, Mutterer J, Ferreux E, Decher G, Schaaf P, Voegel JC, Picart C. Improvement of stability and cell adhesion properties of polyelectrolyte multilayer films by chemical cross-linking. Biomacromolecules 2004;5:284-294 
[59] Dähne L, Leporatti S, Donath E, Möhwald H. Fabrication of micro reaction cages with tailored properties. J Am Chem Soc 2001;123:5431-5436

[60] Petrov AI, Volodkin DV, Sukhorukov GB. Protein-calcium carbonate coprecipitation: a tool for protein encapsulation. Biotechnol Prog 2005;21:918-925

[61] Szarpak A, Cui D, Dubreuil F, De Geest BG, De Cock LJ, Picart C, Auzély-Velty R. Designing hyaluronic acid-based layer-by-layer capsules as a carrier for intracellular drug delivery. Biomacromolecules 2010;11:713-720

[62] Kubisa P. Application of ionic liquids as solvents for polymerization processes. Prog Polym Sci 2004;29:3-12

[63] Erdmenger T, Guerrero-Sanchez C, Vitz J, Hoogenboom R, Schubert U. Recent developments in the utilization of green solvents in polymer chemistry. Chem Soc Rev 2010;39:3317-3333

[64] Kubisa P. Ionic liquids as solvents for polymerization processes - Progress and Challenges. Prog Polym Sci 2009;34:1333-1347

[65] Hong K., Zhang H., Mays J.W., Visser A.E., Brazel C.S., Holbrey J.D., Reichert W.M. and Rogers R.D. Conventional free radical polymerization in room temperature ionic liquids: a green approach to commodity polymers with practical advantages - Chem. Commun., 2002 ; 1368-1369 